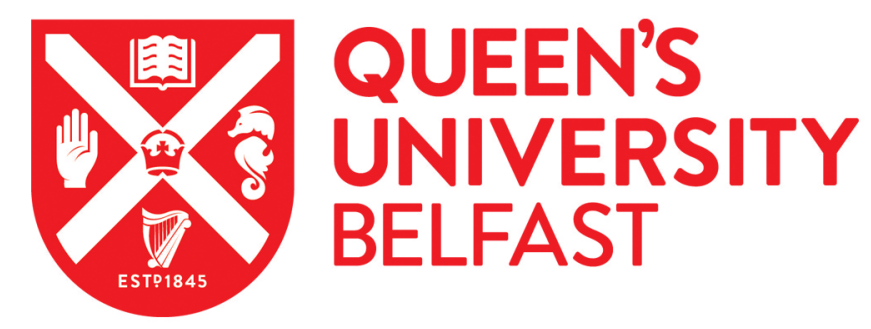

\title{
Size Effects on Thin Film Ferroelectrics: Experiments on Isolated Single Crystal Sheets
}

Chang, L-W., McMillen, M., Morrison, F. D., Scott, J. F., \& Gregg, M. (2008). Size Effects on Thin Film Ferroelectrics: Experiments on Isolated Single Crystal Sheets. Applied Physics Letters, 93(13), [132904]. https://doi.org/10.1063/1.2990760

Published in:

Applied Physics Letters

Queen's University Belfast - Research Portal:

Link to publication record in Queen's University Belfast Research Portal

\section{General rights}

Copyright for the publications made accessible via the Queen's University Belfast Research Portal is retained by the author(s) and / or other copyright owners and it is a condition of accessing these publications that users recognise and abide by the legal requirements associated with these rights.

Take down policy

The Research Portal is Queen's institutional repository that provides access to Queen's research output. Every effort has been made to ensure that content in the Research Portal does not infringe any person's rights, or applicable UK laws. If you discover content in the Research Portal that you believe breaches copyright or violates any law, please contact openaccess@qub.ac.uk. 


\title{
Size effects on thin film ferroelectrics: Experiments on isolated single crystal sheets
}

\author{
L. W. Chang, ${ }^{1}$ M. McMillen, ${ }^{1}$ F. D. Morrison, ${ }^{2}$ J. F. Scott, ${ }^{3}$ and J. M. Gregg ${ }^{1, a)}$ \\ ${ }^{1}$ Centre for Nanostructured Media, School of Maths and Physics, Queen's University Belfast, Belfast BT7 \\ INN, United Kingdom \\ ${ }^{2}$ School of Chemistry, University of St Andrews, North Haugh, St Andrews, Fife KY1 69ST, United Kingdom \\ ${ }^{3}$ Department of Earth Sciences, University of Cambridge, Cambridge CB2 3EQ, United Kingdom
}

(Received 31 July 2008; accepted 8 September 2008; published online 1 October 2008)

\begin{abstract}
Thin lamellae were cut from bulk single crystal $\mathrm{BaTiO}_{3}$ using a focused ion beam microscope. They were then removed and transferred onto single crystal $\mathrm{MgO}$ substrates, so that their functional properties could be measured independent of the original host bulk ferroelectric. The temperature dependence of the capacitance of these isolated single crystal films was found to be strongly bulklike, demonstrating a sharp Curie anomaly, as well as Curie-Weiss behavior. In addition, the sudden change in the remanent polarization as a function of temperature at $T_{C}$ was characteristic of a first order phase change. The work represents a dramatic improvement on that previously published by Saad et al. [J. Phys.: Condens. Matter 16, L451 (2004)], as critical shortcomings in the original specimen geometry, involving potential signal contributions from bulk $\mathrm{BaTiO}_{3}$, have now been obviated. That the functional properties of single crystal thin film lamellae are comparable to bulk, and not like those of conventionally deposited heterogeneous thin film systems, has therefore been confirmed. (C) 2008 American Institute of Physics. [DOI: 10.1063/1.2990760]
\end{abstract}

For decades, there has been a great deal of interest in mapping and understanding changes that occur in the functional characteristics of ferroelectrics as specimen size is reduced. Particular attention has been given to ferroelectric thin films, driven by their potential in memory applications. ${ }^{1,2}$ Establishing fundamental size limits beyond which ferroelectricity can no longer be supported has been a key quest, and has involved careful experimental characterization of ultrathin films, ${ }^{3-5}$ as well as modeling. ${ }^{6}$ However, other changes in the characteristics of ferroelectrics have also been of interest. Since early work by Mead, ${ }^{7}$ an almost universal observation has been that the permittivity of thin film ferroelectrics is progressively and dramatically suppressed as thickness decreases. In most cases, permittivity suppression can be modeled by assuming a parasitic capacitance, of low value, originating at the ferroelectric-electrode boundary and acting electrically in series with "bulklike" ferroelectric material. ${ }^{7-10}$ However, no definitive origin for the proposed dielectric "dead-layer" has yet been discovered. Some researchers have suggested that it is associated with fundamental interface physics, ${ }^{10-15}$ but there are a great number of other hypotheses which are capable of generating series capacitance behavior: strain gradients; ${ }^{16,17}$ physical layers of low permittivity either adjacent to the electrodes in the capacitor $^{18,19}$ or at grain boundaries in the ferroelectric; ${ }^{20}$ parasitic capacitance associated with the finite charge screening lengths in nonideal electrodes; ${ }^{21-24}$ even subtleties in device asymmetry. ${ }^{25}$ Such a variety of possible explanations for permittivity suppression makes for interesting and active debate, but it equally leads to a great deal of confusion and uncertainty, and does not clearly define how detrimental effects on permittivity might be avoided.

Recently, Saad and co-workers ${ }^{26-28}$ have attempted to produce more definitive experimental data than had previ-

\footnotetext{
a) Author to whom correspondence should be addressed. Electronic mail: m.gregg@qub.ac.uk.
}

ously been available, by investigating the functional characteristics of almost freestanding "thin film" capacitors cut directly from single crystals using a focused ion beam microscope (FIB). Films as thin as $\sim 75 \mathrm{~nm}$ appeared to show almost perfect bulklike response, without any signs of permittivity suppression. ${ }^{27}$ The implication was that most previous observations of "dead-layer" interfacial capacitance had resulted from avoidable extrinsic factors, not from fundamentals associated with reduced size or interface physics.

However, a valid criticism of the work was that the thin films were not removed from the bulk single crystal from which they had been machined; rather they were functionally tested in situ, surrounded by bulk material. ${ }^{26,27}$ There was therefore a possibility that the observed bulklike permittivity response was not that from the thin film region at all, and instead resulted from fringing fields sampling the bulk crystal, or from inaccurate subtraction of the background signal from contact pads on the surfaces of the crystals. Given this cause for doubt, there was an imperative to perform further experiments in which the thin film single crystal sheets were tested in isolation. The methodology used and results obtained are described in this letter.

Sheets or lamellae, $\sim 6 \times 13 \mu \mathrm{m}^{2}$ in area and of uniform thickness $(\sim 250-300 \mathrm{~nm})$, were cut from single crystal $\mathrm{BaTiO}_{3}$ using standard automated software on a FEI FIB200TEM FIB. In contrast to previous research, ${ }^{26-28}$ these thin lamellae were cut free from the bulk crystal (using FIB), were removed using a sharp glass needle and micromanipulator, and were then transferred to rest on the surface of a clean $\mathrm{MgO}$ single crystal substrate. Thermal annealing in oxygen to recover ion beam damage and expel gallium ${ }^{26,29}$ was then performed before sputter coating the $\mathrm{BaTiO}_{3}$ lamellae, and $\mathrm{MgO}$ carrier in platinum. FIB machining defined electrodes and contact pads (the interelectrode strip was $\sim 2 \mu \mathrm{m}$ wide). Further annealing was then performed before functional testing. Figure 1 shows a schematic of the process. 


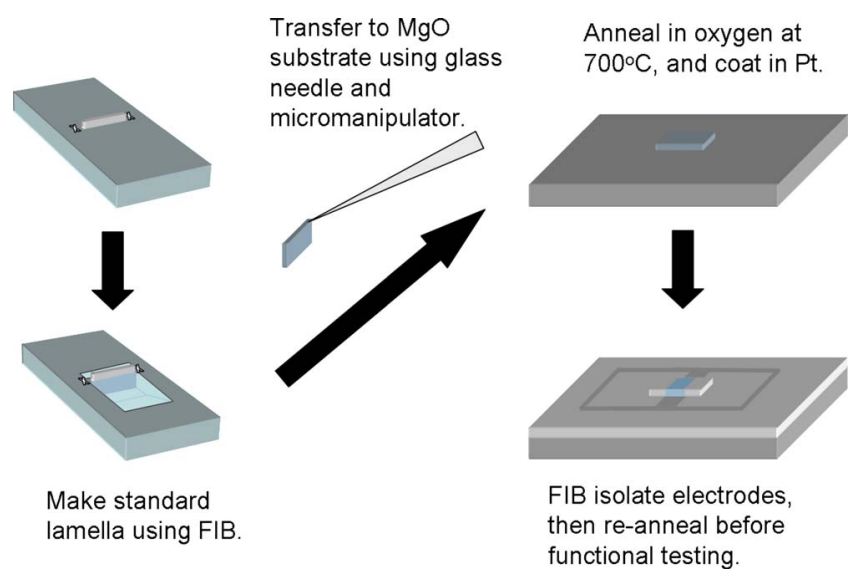

FIG. 1. (Color online) Schematic illustration of the processing steps involved in making test structures to allow the functional characterization of thin lamellae of single crystal $\mathrm{BaTiO}_{3}$, completely isolated from the host bulk crystal from which it had been FIB cut.

The capacitance of the samples was measured as a function of temperature, frequency, and applied voltage $(C-V)$, using micromanipulators to touch the contact pads. The background capacitance contribution from the $\mathrm{MgO}$, and from external circuitry was compensated for by measuring a "dummy" structure in which there was no $\mathrm{BaTiO}_{3}$ sheet. The real capacitor structure and dummy were machined on the same $\mathrm{MgO}$ substrate and mounted on a copper block, which had resistance heaters attached.

Figure 2 shows the temperature dependence of the capacitance, after subtraction of the background. Although data are presented for $100 \mathrm{kHz}$, it should be noted that there was little frequency dispersion in the capacitance. Modeling the behavior as that of a constant phase element ${ }^{30}$ with

$$
\chi^{\prime}(\omega) \propto \omega^{n-1},
$$

where $\chi^{\prime}$ is the real dielectric susceptibility (proportional to the capacitance), and $\omega$ is the angular frequency, gave a value for $n$ close to unity (0.96). As can be seen in Fig. 2, the isolated single crystal $\mathrm{BaTiO}_{3}$ sheet displays a sharp Curie anomaly at approximately the same temperature as bulk barium titanate $(\sim 400 \mathrm{~K})$. Curie-Weiss behavior is evident [Fig. 2(b)], and $T_{0}$ is less than $T_{C}$, characteristic of first order transition behavior.

Such sharp Curie peaks are not seen in conventionally grown thin film ferroelectrics, and, as discussed below, are

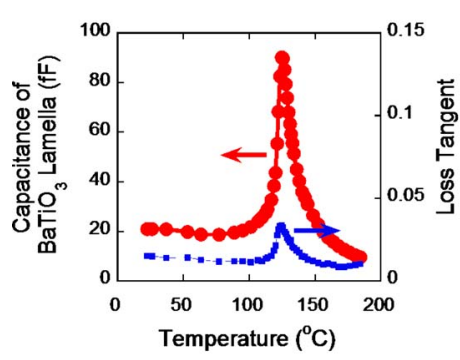

(a)

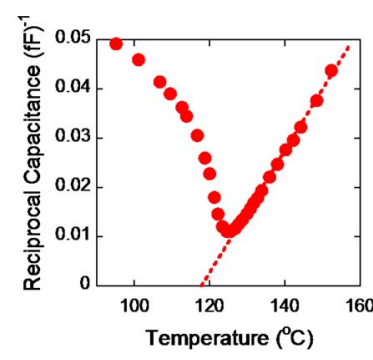

(b)
FIG. 2. (Color online) (a) Capacitance and dielectric loss tangent at $100 \mathrm{kHz}$ attributed to the $\mathrm{BaTiO}_{3}$ lamella. Loss values presented suffered from a lack of sensitivity of the electronic measurement systems used, and are included simply to demonstrate that they are relatively low. The form of the apparent loss peak should not be over interpreted. Curie-Weiss plot (b) taken from the capacitance data in (a). $T_{0}$ is clearly below $T_{C}$.

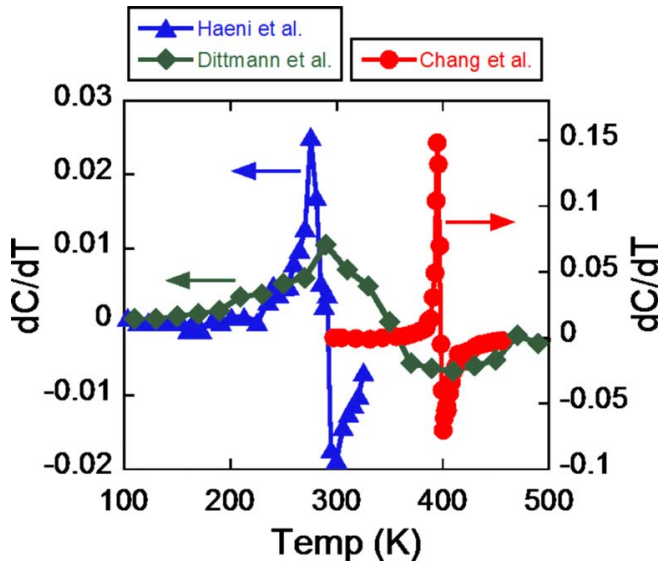

FIG. 3. (Color online) $d C / d T$ (gradient of capacitance with temperature), plotted against temperature as a means of comparing the widths of the Curie anomalies seen in this work with other studies in literature. Prior to making the gradient analysis and comparisons, all data sets were normalized by the peak value of capacitance or permittivity observed in each study.

incommensurate with dead-layer interfacial capacitance. This is because the characteristic series addition of dielectric responses follows the form

$$
1 / C_{T}=\sum_{i} 1 / C_{i},
$$

where $C_{T}$ is the overall measured capacitance, and $C_{i}$ represents the capacitance of the various components which sum electrically in series with each other. Given Eq. (2), the influence of the interfacial capacitance is greatest at the Curie anomaly, and less influential far from the anomaly. This automatically generates a peak smearing effect. Quantification of smearing has been attempted by Parker et al., ${ }^{31}$ fitting peaks to equations such as that originally used by Smolenskii. ${ }^{32}$ Here, we take a different approach, and define the peak width by analyzing the form of the derivative of capacitance with respect to temperature, as a function of temperature (see Fig. 3). We identify the temperature difference $\left(\Delta T_{\text {inflex }}\right)$ between the maximum and minimum points in $d C / d T$ (equivalent to the temperature difference between the two points of inflexion either side of the Curie peak), and use this as a characteristic measure of peak width.

The isolated single crystal thin film lamella, responsible for the data in Fig. 2, demonstrated a $\Delta T_{\text {inflex }}$ value of $6{ }^{\circ} \mathrm{C}$, only slightly larger than that of the bulk single crystal from which it had been machined $\left(2{ }^{\circ} \mathrm{C}\right)$; the uncharacteristically sharp anomaly seen by Haeni et al. ${ }^{33}$ had a $\Delta T_{\text {inflex }}$ value of $\sim 20{ }^{\circ} \mathrm{C}$, while one of the most bulklike thin film responses

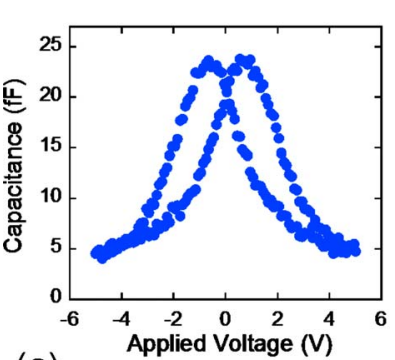

(a)

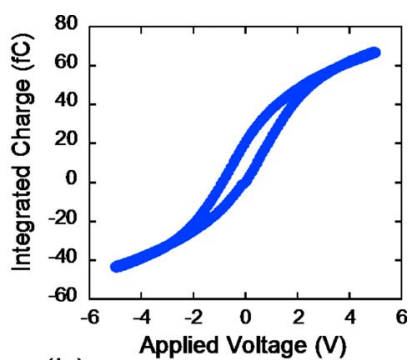

(b)
FIG. 4. (Color online) Capacitance-voltage $(C-V)$ plot taken at $76{ }^{\circ} \mathrm{C}$ (a) at $100 \mathrm{kHz}$; the associated integrated charge (which scales as polarization)voltage plots (b) were constructed from the $C-V$ data. 


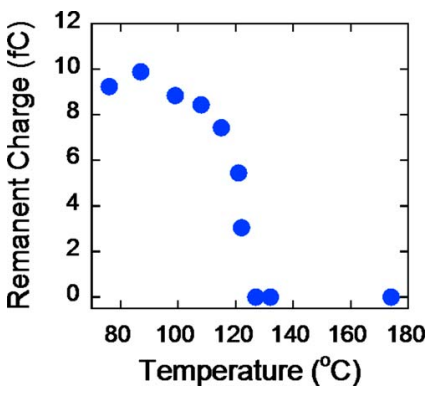

FIG. 5. (Color online) Remanent charge (which scales as remanent polarization) as a function of temperature shows the dramatic collapse at $T_{C}$ that is strongly suggestive of bulklike first order behavior.

seen to date by Dittmann et al. ${ }^{34}$ had a value of $\sim 120{ }^{\circ} \mathrm{C}$. Two other selected data sets typical of conventional thin film behavior published by Yoneda et al. ${ }^{35}$ and Hoerman et al. ${ }^{36}$ had values of $\sim 30$ and $>160{ }^{\circ} \mathrm{C}$, respectively.

Figure 4(a) illustrates typical capacitance-voltage $(C-V)$ data, which were used to reconstruct integrated chargevoltage plots (which scale as the polarization-field, or $P-E$, response). Normally it is thought that $C$ - $V$ data cannot be used to reconstruct charge-voltage loops as

$$
\int C d V=\int \frac{d Q}{d V} d V=Q+K
$$

where the value of $K$ is unknown. Here, however, we have reconstructed the loops numerically by starting at the origin, and assuming linear charge development according to the value of the zero-field capacitance up to a bias of $0.05 \mathrm{~V}$; between 0.05 and $0.15 \mathrm{~V}$, the charge is assumed to further increase linearly according to the capacitance value measured at $0.1 \mathrm{~V}$ bias (the midpoint for the range of assumed applicability). Extension of this numerical reconstruction across all the measured $C-V$ data produced loops of the form illustrated in Fig. 4(b). For analysis of the implied remanent polarization behavior, loops were numerically centered at the origin, and the magnitude of the positive and negative integrated charge values at zero bias voltage averaged. Figure 5 shows the temperature evolution of the zero-field remanent charge (equivalent to remanent polarization). Its sudden collapse on heating at $T_{C}$ again suggests first-order phase transition behavior, typical of bulk, but not seen in conventionally grown thin films. Attempts were made to measure $P-E$ loops directly with a Radiant Precision ferroelectrics characterization unit. However, the magnitudes of the switching currents were below that measurable by the commercial system.

In summary, thin lamellae have been FIB-machined from bulk single crystal $\mathrm{BaTiO}_{3}$, removed from the host crystal and transferred onto a $\mathrm{MgO}$ carrier for functional testing. Capacitance-temperature data show a sharp Curie anomaly around the bulk $T_{C}$, and adherence to Curie-Weiss behavior, yielding $T_{0}$ values lower than $T_{C}$. In addition, charge-voltage loops, reconstructed from capacitancevoltage measurements, suggest a sudden collapse in the remanent polarization at $T_{C}$, consistent with a first order phase transition. These functional characteristics are essentially bulklike, and strongly support observations made in previous work on thin single crystal lamellae that were still attached to their host bulk crystals. ${ }^{27}$ The results add confidence to the view that dead-layer parasitic capacitances are not a general intrinsic feature of thin film ferroelectric behavior.

The authors thank G. Catalan for helpful discussion, and the EPSRC for financial support; F.D.M. would also like to thank the Royal Society for support.

${ }^{1}$ International Technology Roadmap for Semiconductors (ITRS), 2006 Update, 2006 (unpublished), p. 23.

${ }^{2}$ J. F. Scott, Ferroelectric Memories (Springer, Berlin, 2000).

${ }^{3}$ D. D. Fong, G. B. Stephenson, S. K. Streiffer, J. A. Eastman, O. Auciello, P. H. Fuoss, and C. Thompson, Science 304, 1650 (2004).

${ }^{4}$ D. D. Fong, A. M. Kolpak, J. A. Eastman, S. K. Streiffer, P. H. Fuoss, G. B. Stephenson, C. Thompson, D. M. Kim, K. J. Choi, C. B. Eom, I. Grinberg, and A. M. Rappe, Phys. Rev. Lett. 96, 127601 (2006).

${ }^{5}$ S. K. Streiffer, J. A. Eastman, D. D. Fong, C. Thompson, A. Munkholm, M. V. R. Murty, O. Auciello, G. R. Bai, and G. B. Stephenson, Phys. Rev. Lett. 89, 067601 (2002).

${ }^{6}$ J. Junquera and P. Ghosez, Nature (London) 422, 506 (2003).

${ }^{7}$ C. A. Mead, Phys. Rev. Lett. 6, 545 (1961).

${ }^{8}$ L. J. Sinnamon, R. M. Bowman, and J. M. Gregg, Appl. Phys. Lett. 78, 1724 (2001)

${ }^{9}$ C. Basceri, S. K. Streiffer, A. I. Kingon, and R. Waser, J. Appl. Phys. 82, 2497 (1997).

${ }^{10}$ O. G. Vendik and S. P. Zubko, J. Appl. Phys. 88, 5343 (2000).

${ }^{11}$ M. Stengel and N. A. Spaldin, Nature (London) 443, 679 (2006).

${ }^{12}$ C. Zhou and D. M. Newns, J. Appl. Phys. 82, 3081 (1997).

${ }^{13}$ K. Natori, D. Otani, and N. Sano, Appl. Phys. Lett. 73, 632 (1998).

${ }^{14}$ O. G. Vendik, S. P. Zubko, and L. T. Ter-Martirosayn, Appl. Phys. Lett. 73, 37 (1998).

${ }^{15}$ M. Tyunina and J. Levoska, Appl. Phys. Lett. 88, 262904 (2006).

${ }^{16}$ G. Catalan, L. J. Sinnamon, and J. M. Gregg, J. Phys.: Condens. Matter 16, 2253 (2004).

${ }^{17}$ G. Catalan, B. Noheda, J. McAneney, L. J. Sinnamon, and J. M. Gregg, Phys. Rev. B 72, 020102 (2005).

${ }^{18}$ I. Stolichnov, A. Tagantsev, N. Setter, J. S. Cross, and M. Tsukada, Appl. Phys. Lett. 75, 1790 (1999).

${ }^{19}$ D. Choi, B. Kim, S. Son, S. Oh, and K. Park, J. Appl. Phys. 86, 3347 (1999).

${ }^{20}$ L. J. Sinnamon, M. M. Saad, R. M. Bowman, and J. M. Gregg, Appl. Phys. Lett. 81, 703 (2002).

${ }^{21}$ M. Dawber and J. F. Scott, Jpn. J. Appl. Phys., Part 1 41, 6848 (2002).

${ }^{22}$ C. T. Black and J. J. Welser, IEEE Trans. Electron Devices 46, 776 (1999).

${ }^{23}$ H. Y. Ku and F. G. Ullman, J. Appl. Phys. 35, 265 (1964).

${ }^{24}$ G. Gerra, A. K. Tagantsev, N. Setter, and K. Parlinski, Phys. Rev. Lett. 96, 107603 (2006).

${ }^{25}$ A. M. Bratkovsky and A. P. Levanyuk, Phys. Rev. Lett. 94, 107601 (2005)

${ }^{26}$ M. M. Saad, R. M. Bowman, and J. M. Gregg, Appl. Phys. Lett. 84, 1159 (2004).

${ }^{27}$ M. M. Saad, P. Baxter, R. M. Bowman, J. M. Gregg, F. D. Morrison, and J. F. Scott, J. Phys.: Condens. Matter 16, L451 (2004).

${ }^{28}$ M. M. Saad, P. Baxter, J. McAneney, A. Lookman, L. J. Sinnamon, P. Evans, A. Schilling, T. Adams, X. Zhu, R. J. Pollard, R. M. Bowman, J. M. Gregg, P. Zubko, D. J. Jung, F. D. Morrison, and J. F. Scott, IEEE Trans. Ultrason. Eng. 53, 2208 (2006).

${ }^{29}$ A. Schilling, T. Adams, R. M. Bowman, and J. M. Gregg, Nanotechnology 18, 035301 (2007).

${ }^{30}$ F. D. Morrison, D. J. Jung, and J. F. Scott, J. Appl. Phys. 101, 094112 (2007).

${ }^{31}$ C. B. Parker, J. P. Maria, and A. I. Kingon, Appl. Phys. Lett. 81, 340 (2002).

${ }^{32}$ G. A. Smolenski, J. Phys. Soc. Jpn. S28, 26 (1970).

${ }^{33}$ J. H. Haeni, P. Irvin, W. Chang, R. Uecker, P. Reiche, Y. L. Li, S. Choudhury, W. Tian, M. E. Hawley, B. Craigo, A. K. Tagantsev, X. Q. Pan, S. K. Streiffer, L. Q. Chen, S. W. Kirchoefer, J. Levy, and D. G. Schlom, Nature (London) 430, 758 (2004).

${ }^{34}$ R. Dittmann, R. Plonka, E. Vasco, N. A. Pertsev, J. Q. He, C. L. Jia, S Hoffmann-Eifert, and R. Waser, Appl. Phys. Lett. 83, 5011 (2003).

${ }^{35}$ Y. Yoneda, K. Kasatani, H. Terauchi, Y. Yano, T. Terashima, and Y. Bando, J. Phys. Soc. Jpn. 62, 1840 (1993).

${ }^{36}$ B. H. Hoerman, G. M. Ford, L. D. Kaufmann, and B. W. Wessels, Appl. Phys. Lett. 73, 2248 (1998). 